\title{
Tendência secular em estatura em recrutas da Marinha do Brasil nascidos entre 1940 e 1965
}

\author{
Secular trends in the stature of Brazilian Navy \\ recruits born from 1940 to 1965
}

Gilberto Kac 1

\footnotetext{
1 Departamento de Nutrição Social e Aplicada, Instituto de Nutrição Josué de Castro, Universidade Federal do Rio de Janeiro. Rua Brigadeiro Trompowsky s/no, Bloco J, 20 andar, Rio de Janeiro, RJ 21941-590, Brasil.
}

Abstract The present paper reports data on secular trends in the stature of Brazilian Navy recruits born from 1940 to 1965. The final sample included 3269 individuals aged 18.00-18.99. Statistics performed were: Anova (one-way and two-way), Sheffe test, si mplelinear regression between stature and year of birth, and multiple linear regression adjusting for level of schooling (beta coefficient) and chi-square. Results indicated a progressive growth trend in stature of 0.1 $\mathrm{cm} / \mathrm{yr}$. for the country as a whole. The trend was al so observed for nearly all regions and two out of three levels of schooling and can be explained by improvement in some of the country's health indicators. One important characteristic was a higher level of schooling observed among Navy recruits, suggesting that these individuals represent a highly sel ect group, and that therefore data on the Navy cannot be applied directly to the Brazilian population as a whole.

Key words Body Height; Anthropometry; Nutrition; Military Personnel

Resumo O presente artigo apresenta dados sobre tendência secular em estatura em recrutas da Marinha do Brasil nascidos no período 1940-1965. A amostra final constituiu-se de 3.269 indivíduos com idade entre 18,00-18,99 anos. Foram realizadas as análi ses estatísticas: Anova (oneway e two-way), teste de Sheffe, regressão simples entre estatura e ano de nascimento e múlti pla, controlando para escolaridade (coefici ente angular), e Qui-Quadrado. Os resultados demonstraram existência de um aumento progressi vo em estatura da ordem de $0,1 \mathrm{~cm} /$ ano para o conjunto das regiões do País. Também se verificou tendência posi tiva em quase todas regi ões e estratos de escolaridade. Em nível nacional, este aumento pode ser atribuído a mel horias em algunsindicadores sanitários. Argumenta-se que os resultados não podem ser diretamente extrapolados para a população brasileira como um todo, uma vez que os jovens que sealistam na Marinha apresentam um perfil antropométrico e sócio-econômi co di ferenciado daquele da população geral . Palavras-chave Estatura; Antropometria; Nutrição; Militares 


\section{Introdução}

Tendência secular em estatura pode ser definida como a ocorrência de mudanças na idade em que se atinge uma determinada estatura ou modificações na estatura adulta final de uma população (Van Wieringen, 1986). Louis Villermé pode ser considerado pioneiro na realização de análises sobre ten dência secular em estatura em razão de seus estudos sobre o crescimento físico de recrutas franceses no início do século XIX. Por meio deles demonstrou que são grandes as influências exercidas pelo meio ambiente na estatura final alcançada por uma população (Villermé, 1829 apud Tanner, 1981:162).

O fenômeno de tendência secular é relevante, tanto por razões práticas, como teóricas. Deve-se estar atento, por exemplo, ao efeito que o mesmo pode exercer na utilização de curvas de crescimento para avaliação do estado nutricional (Garn, 1987). Uma outra abordagem é a que permite, com base em séries históricas de estatura, caracterizar mudanças nos padrões econômicos e de qualidade de vida de populações (Fogel et al., 1982; Tanner, 1982; Floud et al., 1990).

A ocorrência de tendência secular em estatura está bem documentada para a Europa, Estados Unidos e Japão (Tanner, 1966; Greulich, 1976; Roche, 1979; Fogel et al., 1983; Eveleth \& Tanner, 1990; Floud et al., 1990; Malina, 1990; Greiner \& Gordon, 1992). Os progressivos aumentos observados nas médias de estatura são atribuídos de forma geral a melhorias nas condições ambientais, incluindo maior acesso a serviços de saúde, maior disponibilidade de alimentos, melhoria de condições sócio-econômicas, entre outros (Fogel, 1986; Van Wieringen, 1986).

No Brasil, são escassos os estudos enfocando a evolução da estatura adulta baseados em séries temporais. Dados provenientes de instituições militares têm sido os mais freqüentemente utilizados para caracterizar este tipo de tendência (Victora et al., 1989; M arcondes \& Marques, 1993; Figueiró, 1994; Paiva, 1994). No entanto, a maioria dos estudos enfocando séries históricas restringe-se a regiões específicas do País. Recentemente, alguns autores têm procurado compensar a escassez de trabalhos sobre tendência secular através de análises baseadas em estudos transversais de representatividade nacional (Monteiro et al., 1994, 1995).

$O$ presente artigo analisa a ten dência secular em estatura de recrutas da Marinha Brasileira nascidos entre 1940 e 1965, complementando análises de dados mais contemporâneos apresentados em outro trabalho (Kac \& Santos,
1997). São feitas também considerações teórico-metodológicas sobre a utilização de bancos de dados antropométricos provenientes de instituições militares visando a caracterização de tendência secular em estatura.

\section{Material e métodos}

A análise foi realizada tendo como referência um banco de dados contendo as seguintes variáveis: distrito e data de recrutamento, data e local de nascimento, estatura em centímetros (sem precisão decimal) e escolaridade. Os dados foram coletados de um arquivo localizado na Diretoria de Pessoal Militar da Marinha (DPM M), situada na cidade do Rio de Janeiro, que centraliza informações dos recrutados de todos os seis Distritos Navais do Brasil. A idade foi calculada, valendo-se das variáveis data de recrutamento e data de nascimento. A variável local de nascimento, disponível segundo a Unidade da Federação, foi agrupada de acordo com as regiões. Como o universo não pôde ser precisamente estimado, objetivou-se al cançar uma amostra de $10 \%$ do total de registros. Para tanto, sistematicamente, de cada dez fichas de recrutamento, foram coletados dados da décima. O total de registros coletados foi de 6.758 . A se julgar pelos dados analisados, grande parte dos recrutados pela Marinha provêm das regiões Sudeste $(28,8 \%)$ e Nordeste $(31,7 \%)$, o que foi também observado na análise de dados contemporâneos conduzida por Kac \& Santos (1997).

Com relação à idade, a grande maioria dos 6.758 registros de recrutados era de indivíduos entre 17 e 21 anos de idade $(96,4 \%)$, com uma concentração em 18 (48,4\%) e 19 anos (22,4\%) (indivíduos recrutados tardiamente). Estes dados estão de acordo com os critérios de alistamento da Marinha, que indicam que os rapazes devem se alistar no ano em que completam 18 anos. Havia no banco de dados uma pequena quantidade de indivíduos com idade menor que 17 anos (1,1\%), parcela significativa da qual deve estar associada a erro de coleta das datas de nascimento. Havia também uma porcentagem baixa de indivíduos com idade maior que 21 anos (2,5\%), que deve se referir a jovens que se alistam tardiamente.

Para fins desta análise, optou-se por trabaIhar somente com indivíduos com idade entre 18,00 e 18,99 anos e aqueles nascidos entre 1940 e 1965 . Foram excluídos por estes critérios $3.473(51,4 \%)$ e 16 casos $(0,2 \%)$, respectivamente. Contrário a Kac \& Santos (1997), no presente artigo não foi possível controlar para a 
questão da mobilidade populacional (manutenção no banco de dados somente dos indivíduos que nasceram e foram recrutados numa mesma região), o que reduziria ainda mais o tamanho da amostra. Restaram, portanto, 3.269 indivíduos, o que correspondeu a 48,4\% da amostra coletada inicialmente. As análises foram realizadas segundo qüinqüênio de ano de nascimento, quais sejam: 1940-44, 1945-49, 1950-54, 1955-59 e 1960-65.

Dentre as variáveis presentes no banco, a escolaridade, transformada em número de anos de instrução, é a mais estreitamente associada à condição sócio-econômica. Esta variável foi agrupada em três categorias: 1-4; 5-8 e 9-11 anos de instrução. Foram eliminados 177 casos $(5,4 \%)$ em razão da falta de informação de escolaridade.

Não foi possível obter informações detalhadas acerca dos procedimentos empregados na coleta dos dados de estatura. Nas fichas, os vaIores de estatura estão registrados em números inteiros. Visando investigar a presença de viés de aferição, analisaram-se as freqüências de valores do último dígito de estatura ao longo do período. Para tanto, inicialmente foi realizado um teste de aderência à distribuição uniforme, a fim de verificar a existência de concentração em algum dígito. Em seguida, testou-se, através do Qui-Quadrado (comparação entre proporções), se elas se mantiveram constantes ao longo da série.

As taxas de ganho médio em estatura ao longo dos qüinqüênios foram calculadas valendo-se dos coeficientes angulares gerados com base em modelos de regressão linear, que tinham como variável dependente a estatura e independente o ano de nascimento. Foram realizadas análises de variância (one-way) entre estatura e qüinqüênio de nascimento, teste de Scheffe para identificar diferenças entre médias, Anova (two-way), visando identificar diferenças nas médias de estatura a cada período de cinco anos entre as regiões, e a interação entre estas duas variáveis na evolução do número de anos de instrução. Foram desenvolvidas ainda análises de regressão múltipla, tendo como variável dependente a estatura e independentes o ano de nascimento e o número de anos de instrução, com o objetivo de investigar o efeito da escolaridade na ocorrência da tendência secular em estatura.

As análises estatísticas foram conduzidas com os pacotes SPSS/ PC + (Marija, 1992) e EpiInfo 6.01 (Dean et al., 1995).

\section{Resultados}

As análises sobre a existência de viés de aferição demonstraram uma concentração nas proporções do último dígito de estatura terminados em 0 e 5 ( $\chi^{2}=38,8$; g.l. $\left.=9 ; p \varangle 0,000012\right)$. Quando se compararam as proporções de cada dígito ao longo dos qüinqüênios pelo teste do Qui-Quadrado, não foram detectadas diferenças estatisticamente significantes. Os resultados das análises sugerem que, mesmo estando presente, o viés de aferição distribuiu-se de forma relativamente homogênea ao longo da série.

A tendência das médias de estatura para o Brasil como um todo demonstrou que ocorreu um aumento da ordem de $0,105 \mathrm{~cm} /$ ano no intervalo de 25 anos ( $p \varangle 0,01)$. A evolução da estatura ao longo desses anos apresentou intensidades distintas de acordo com o qüinqüênio (Figura 1). No decorrer da primeira metade da década de 40 , houve um declínio na média de estatura de $1,1 \mathrm{~cm}$. A partir da segunda metade dessa mesma década, a tendência foi de aumento. O teste de Scheffe indicou que a média para o último qüinqüênio diferiu das demais e os resultados da análise de variância one way demonstraram a existência de diferenças entre as médias de estatura ao longo dos qüinqüênios.

A Tabela 1 apresenta valores de estatura média ao longo do período segundo qüinqüênios de nascimento para as cinco regiões do País e para o Brasil em geral. Observaram-se aumentos significantes nas médias para todas

Figura 1

Evolução da estatura média de recrutas da Marinha segundo qüinqüênio de nascimento. Brasil, 1940 a 1965.

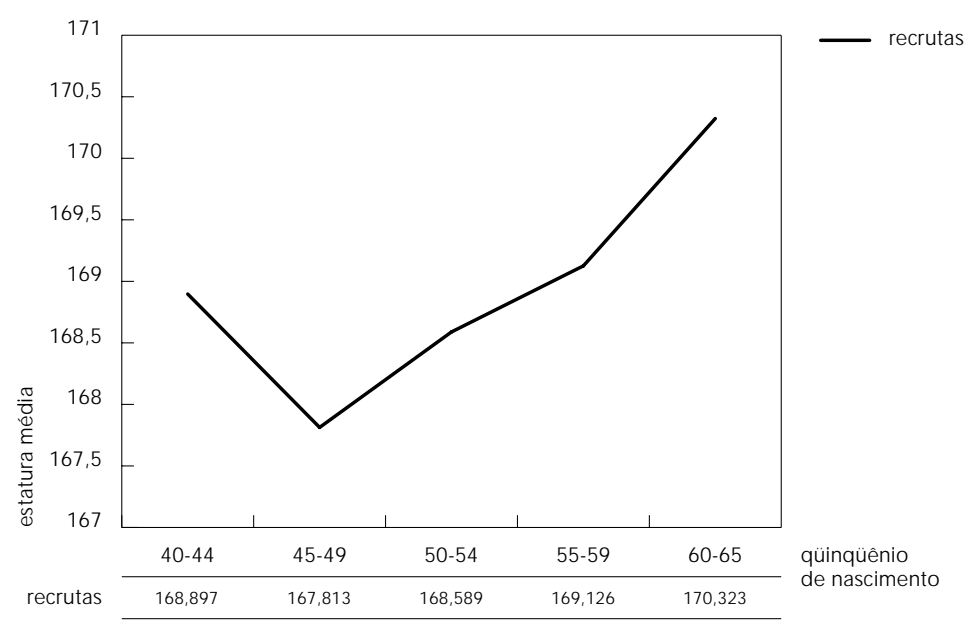


Tabela 1

Evolução da estatura média de recrutas da Marinha segundo qüinqüênio de nascimento e região. Brasil, 1940 a 1965

\begin{tabular}{|c|c|c|c|}
\hline Região & Amostra & Média & D. P. \\
\hline \multicolumn{4}{|l|}{ Sul } \\
\hline $40-44$ & 26 & 170,4 & 5,7 \\
\hline $45-49$ & 22 & 169,4 & 5,1 \\
\hline $50-54$ & 94 & 169,1 & 6,0 \\
\hline $55-59$ & 132 & 170,0 & 6,2 \\
\hline $60-65$ & 130 & 171,6 & 5,6 \\
\hline \multicolumn{4}{|l|}{ Sudeste } \\
\hline $40-44$ & 66 & 169,6 & 5,6 \\
\hline $45-49$ & 47 & 170,6 & 6,2 \\
\hline $50-54$ & 249 & 169,7 & 5,9 \\
\hline $55-59$ & 328 & 170,9 & 6,2 \\
\hline $60-65$ & 252 & 171,4 & 6,5 \\
\hline \multicolumn{4}{|c|}{ Centro-O este } \\
\hline $40-44$ & 21 & 168,4 & 6,2 \\
\hline $45-49$ & 18 & 167,3 & 5,4 \\
\hline $50-54$ & 73 & 169,3 & 6,1 \\
\hline $55-59$ & 84 & 169,9 & 6,0 \\
\hline $60-65$ & 154 & 171,3 & 6,0 \\
\hline \multicolumn{4}{|c|}{ Nordeste } \\
\hline $40-44$ & 109 & 168,4 & 5,6 \\
\hline $45-49$ & 86 & 167,0 & 5,1 \\
\hline $50-54$ & 268 & 168,3 & 5,7 \\
\hline $55-59$ & 303 & 168,4 & 5,8 \\
\hline $60-65$ & 269 & 169,0 & 6,2 \\
\hline \multicolumn{4}{|l|}{ Norte } \\
\hline $40-44$ & 10 & 166,4 & 5,0 \\
\hline $45-49$ & 30 & 165,0 & 6,1 \\
\hline $50-54$ & 155 & 166,6 & 5,2 \\
\hline $55-59$ & 190 & 166,3 & 6,0 \\
\hline $60-65$ & 153 & 168,9 & 6,2 \\
\hline \multicolumn{4}{|l|}{ Brasil } \\
\hline $40-44$ & 232 & 168,9 & 5,6 \\
\hline $45-49$ & 203 & 167,8 & 5,8 \\
\hline $50-54$ & 839 & 168,6 & 5,8 \\
\hline $55-59$ & 1037 & 169,1 & 6,3 \\
\hline $60-65$ & 958 & 170,3 & 6,3 \\
\hline
\end{tabular}

as regiões, com exceção do Nordeste. Também verificou-se um declínio nas médias de estatura na passagem do primeiro para o segundo qüinqüênio em todas as regiões, com exceção do Sudeste. A retomada da média de estatura verificada no início do estudo ocorreu no qüinqüênio 50-54 para as regiões Nordeste, CentroOeste e Norte, enquanto, para a região Sul, o mesmo ocorreu apenas no qüinqüênio 55-59. É justamente na passagem deste qüinqüênio para o último que ocorreram os maiores ganhos na estatura média na região Sul $(1,6 \mathrm{~cm})$, Centro-Oeste $(1,4 \mathrm{~cm})$ e Norte $(2,6 \mathrm{~cm})$. As magnitudes dos valores do coeficiente angular observados para estas regiões foram, portanto, extremamente influenciados pelos ganhos nesse período. As taxas de ganho médio calculadas com base no coeficiente angular demonstraram que o Norte $(0,179 \mathrm{~cm} /$ ano; $p \varangle 0,01)$ e o Centro-Oeste $(0,179 \mathrm{~cm} /$ ano; $p \varangle 0,01)$ foram as regiões que apresentaram as maiores taxas de ganho, seguidas da região Sul $(0,120 \mathrm{~cm} /$ ano; $p<0,05)$ e Sudeste $(0,110 \mathrm{~cm} /$ ano; $p<0,01$ ) (Tabela 2).

Quando as médias de estatura foram comparadas por região, percebeu-se claramente dois grupos distintos, sobretudo a partir do qüinqüênio 50-54: o primeiro, composto pelas regiões mais desenvolvidas (Sul, Sudeste e Centro-Oeste), apresentou sistematicamente médias mais el evadas que o segundo grupo, que inclui o Norte e o Nordeste, as duas regiões economicamente menos desenvolvidas do País (Figura 2). As diferenças nas médias de estatura entre as regiões foram estatisticamente significantes ao longo dos qüinqüênios, como revelaram os resultados da análise de variância twoway. Não foi identificada interação significante entre os efeitos principais.

Persiste a tendência secular em estatura após estratificação por escolaridade (Figura 3). Mesmo não tendo sido detectado um aumento estatisticamente significante em estatura no grupo de mais baixa instrução (coeficiente angular =0,03; $p>0,05$ ), as evidências são claras para as demais categorias. Os indivíduos com cinco a oito anos de instrução apresentaram incremento médio de $0,121 \mathrm{~cm} /$ ano $(p \varangle 0,01)$, enquanto que para os com nove a 11 anos o incremento foi um pouco maior (coeficiente angular =0,149 cm/ano; $p \varangle 0,05$ ).

Buscou-se avaliar a possibilidade de a tendência positiva de aumento em estatura estar associada em parte ao aumento nos níveis de escolaridade. Isto porque o número médio de anos de instrução de recrutados da Marinha aumentou de 3,8 para 8,1, no Brasil, entre 1940 e 1965. Os aumentos em anos de escolaridade 
segundo região foram bastante distintos ao longo do período (Tabela 3). As médias mais do que dobraram nas regiões Sudeste e Nordeste. É interessante observar que o Norte apresentou as maiores médias de escolaridade, sempre superiores às verificadas para o Brasil como um todo. Não obstante, as médias de estatura para esta região estão entre as menores do País. Outra observação que confirma essa tendência de aumento na escolaridade foi a inversão nas proporções entre as categorias de escolaridade. Aqueles com um a quatro anos de instrução representavam $81 \%$ em $1940-44$ e apenas $9,6 \%$ em 1960-65; já os recrutas com cinco a oito anos de instrução aumentaram sua participação de 17,5 para $65,2 \%$ no mesmo período. Chama atenção, ainda, o fato de que há consistência entre os níveis de escolaridade no período 1940-65 e aqueles da década de 70 (Kac \& Santos, 1997).

Análises de regressão múltipla tendo como variável dependente a estatura e como variáveis independentes o número de anos de instrução e ano de nascimento demonstram que ocorreu uma redução da ordem de $27 \%$ após ajuste para escolaridade para o Brasil como um todo (Tabela 2). As taxas de ganho de estatura mantêm-se, no entanto, positivas e estatisticamente significantes (coeficiente angular = 0,$076 ; p<0,01$ ). Depreende-se, conseqüentemente, que o ganho em estatura está associado ao recrutamento de jovens de crescente nível sócio-econômico. Entre as regiões, os resultados das análises de regressão múltipla demonstraram que, após controlada a escolaridade, a tendência secular desapareceu no Sul e Sudeste. No Centro-Oeste e Norte, os coeficientes ajustados foram 38,8\% e 30,2\% inferiores aos não ajustados, respectivamente (Tabela 2 ).

Também foram realizadas análises de regressão excluindo-se o primeiro qüinqüênio, em razão da queda observada na estatura média durante o período no qual estava em curso a Segunda Guerra Mundial (Tabela 2). Como esperado, as taxas de ganho tornaram-se mais pronunciadas segundo região e escolaridade. Os aumentos variaram de $10,9 \%$ no Norte a $40,6 \%$ no Sul. Para o Brasil, o aumento foi de $34,6 \%$. Na região Nordeste, com a exclusão do primeiro qüinqüênio, a taxa de ganho passou a ser estatisticamente significante (coeficiente angular $=0,08 \mathrm{~cm} /$ ano; $\mathrm{p}<0,05$ ). O mesmo ocorreu para os indivíduos de mais baixa escolaridade (coeficiente angular $=0,120 ; p \varangle 0,05$ ). As análises de regressão específicas para o período 1940 a 1944, para o Brasil e segundo regiões, demonstraram que neste qüinqüênio os valores do coeficiente angular foram negativos,
Tabela 2

Coeficientes angulares ajustados e não ajustados, com e sem o primeiro qüinqüênio de nascimento, da regressão estatura, ano de nascimento e escolaridade, para recrutas da Marinha. Brasil, 1940-1965.

\begin{tabular}{llllll}
\hline Região & \multicolumn{2}{c}{1940 a 1965} & 1940 a 1944 & \multicolumn{2}{c}{1945 a 1965} \\
& C. A. & $\begin{array}{c}\text { C. A. } \\
\text { Ajustado }\end{array}$ & C. A. & C. A. & $\begin{array}{c}\text { C. A. } \\
\text { Ajustado }\end{array}$ \\
\hline Sul & $0,120^{*}$ & $0,09 \mathrm{~ns}$ & $-0,617 \mathrm{~ns}$ & $0,202^{*}$ & $0,189 * *$ \\
Sudeste & $0,110^{*}$ & $0,05 \mathrm{~ns}$ & $0,493 \mathrm{~ns}$ & $0,134^{* *}$ & $0,06 \mathrm{~ns}$ \\
Centro-Oeste & $0,179 *$ & $0,129 * *$ & $-2,61 * *$ & $0,214^{*}$ & $0,178^{* *}$ \\
Nordeste & $0,04 \mathrm{~ns}$ & $0,005 \mathrm{~ns}$ & $0,08 \mathrm{~ns}$ & $0,08^{* *}$ & $0,04 \mathrm{~ns}$ \\
Norte & $0,179 *$ & $0,125^{* *}$ & $-0,677 \mathrm{~ns}$ & $0,201^{*}$ & $0,151^{* *}$ \\
Brasil & $0,105^{*}$ & $0,076^{*}$ & $-0,03 \mathrm{~ns}$ & $0,159 *$ & $0,131^{*}$ \\
\hline
\end{tabular}

C. A. = Coeficiente angular.

C. A. Ajustado $=$ Coeficiente angular ajustado pela escolaridade.

$* p<0,01$

$* * p<0,05$

Figura 2

Evolução da estatura média de recrutas da Marinha segundo qüinqüênio de nascimento e região. Brasil, 1940 a 1965.

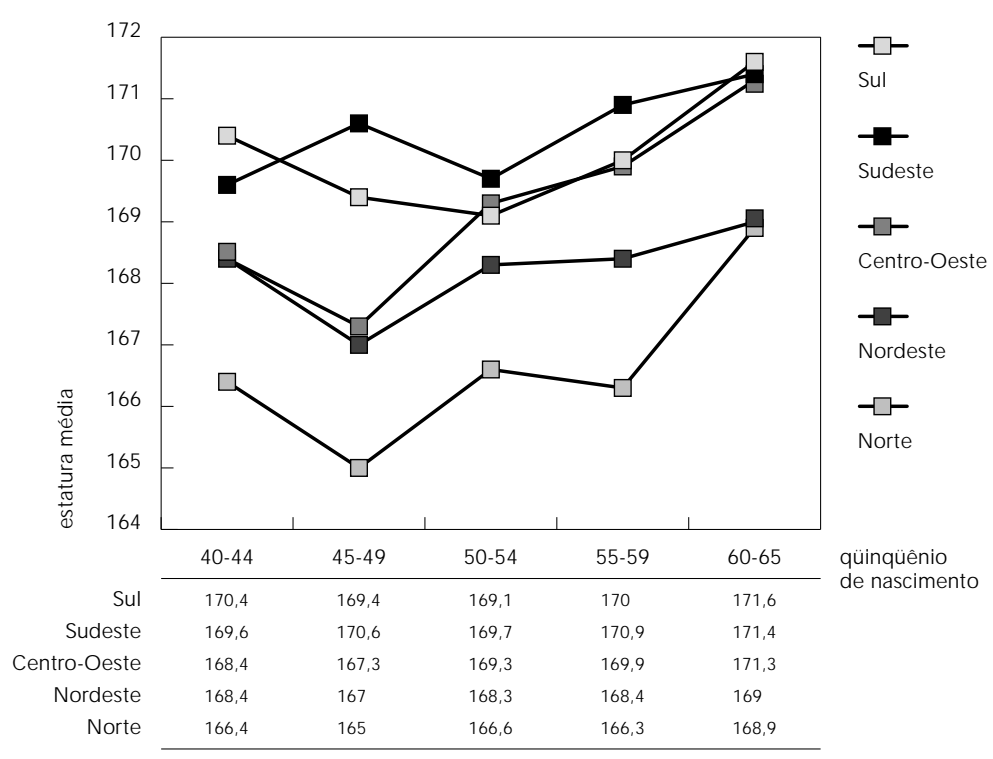


Figura 3

Evolução da estatura média de recrutas da Marinha segundo qüinqüênio de nascimento e escolaridade. Brasil, 1940 a 1965.

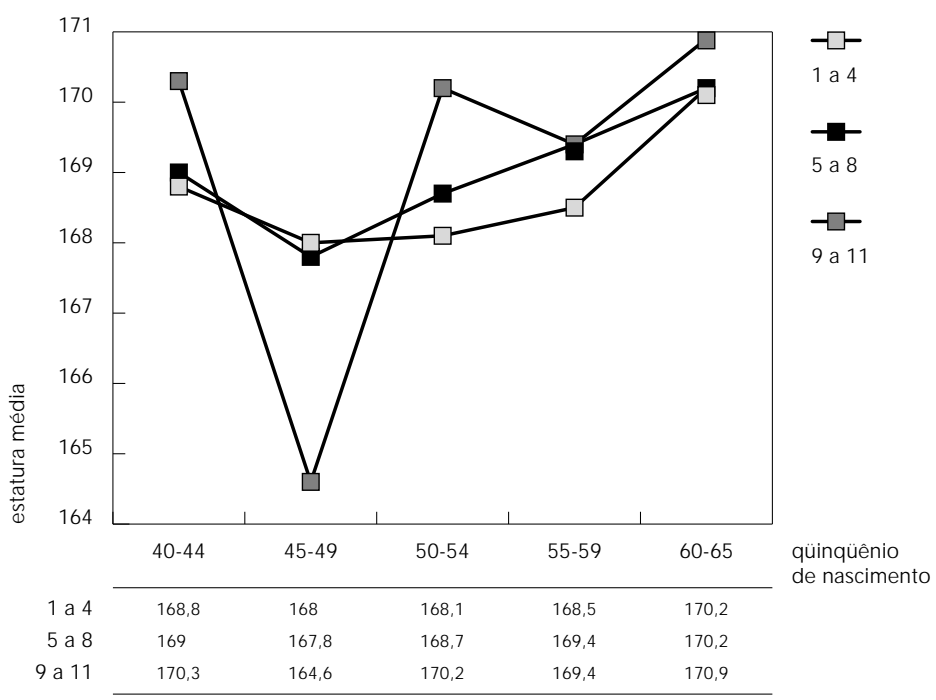

com exceção do Nordeste e do Sudeste, que não apresentaram declínio na estatura média. Estes valores, contudo, só foram estatisticamente significantes para a região Centro-Oeste.

As análises de regressão múltipla sem o primeiro qüinqüênio (controlando para escolaridade) evidenciaram que no Sudeste e no Nordeste a tendência desapareceu, enquanto no Sul (coeficiente angular $=0,189 ; p<0,05$ ), Centro-Oeste (coeficiente angular $=0,178 ; p<0,05$ ) e no Norte (coeficiente angular $=0,152 ; p<0,05$ ) a tendência manteve-se presente.

\section{Discussão}

Os resultados deste trabalho demonstram incontestavelmente a ocorrência de tendência secular em estatura a partir da segunda metade da década de 40 deste século no Brasil.

É interessante observar que os incrementos alcançados neste período são bem menores do que aqueles obtidos para alistados e recrutados nascidos na década de 70 (Kac \& Santos, 1997). Estas diferenças podem ser explicadas pelo fato de que melhorias significantes no desenvolvimento sanitário refletiram na estatura somente a partir da década de 60 . Com relação à mortalidade infantil, por exemplo, Simões \& Monteiro (1995) demonstraram ter sido intenso o declínio ( $20 \%$ a $30 \%$ ) para este indicador entre os anos 40 e 50. Se a queda da mortalidade foi particularmente pronunciada a partir da década de 40, esperar-se-ia que seus efeitos sobre a estatura adulta se manifestassem duas décadas depois. De fato, verificaram-se, neste estudo, el evadas taxas de ganho em estatura neste período, como demonstraram os coeficientes angulares gerados a partir de regressões com o qüinqüênio 1960-1965 (coeficiente angular $=0,236 \mathrm{~cm} /$ ano; $p<0,05$ ). Ademais, segundo o teste de Sheffe, a única média de estatura que difere em todo o período é a do último qüinqüênio.

Uma ressalva a ser feita sobre a utilização de dados de estatura originários de bancos de dados militares visando à caracterização de tendência secular diz respeito à seletividade da amostra. O rigoroso processo de seleção implementado pelas Forças Armadas torna, sem dúvida, o grupo para qual as análises foram realizadas muito seletivo e, conseqüentemente, inapropriado para a realização de inferências populacionais para o Brasil como um todo. Um exemplo característico é o da região Norte do País, na qual verificou-se a mais elevada média de anos de instrução, sugerindo que apenas os indivíduos de maior escolaridade seriam selecionados. No que tange à totalidade das regiões, esta é reconhecida como a de menor escolaridade (IBGE, 1963).

Outro ponto que deve ser considerado diz respeito às inter-relações entre o aumento de escolaridade influenciando o aumento médio de estatura. De fato, os resultados das análises de regressão múltipla demonstraram que a tendência desapareceu em algumas regiões após o controle para a escolaridade. Assim, nestas regiões, o aumento em estatura pode ser atribuído ao recrutamento de indivíduos com cada vez mais escolaridade e, por conseqüência, à incorporação de recrutas com maiores níveis de instrução.

O resultados reportados por Monteiro et al. (1994, 1995), que compararam duas coortes nascidas em 1952 e 1967 com idade de 22 anos, apontam para um ganho de $1,3 \mathrm{~cm}(0,09 \mathrm{~cm} /$ ano), diferenciados sócio-economicamente. No período entre 1952 e 1965, a taxa média observada para os recrutados da Marinha foi de $0,166 \mathrm{~cm} /$ ano $(p<0,01)$, o que reforça a idéia de que estes jovens apresentam um perfil antropométrico diferenciado do da população geral, já que os dados reportados por Monteiro et al. (1994, 1995) provêm de bancos de dados de representatividade nacional.

O incremento médio observado neste trabalho para os recrutas da Marinha na região Sul do País foram comparados com os reportados por Victora et al. (1989), para os recrutas do Exército. O que se observou foi que a taxa mé- 


\section{Tabela 3}

Evolução do número médio de anos de estudo para recrutados pela Marinha segundo qüinqüênio de nascimento e região. Brasil, 1940-1965.

\begin{tabular}{|c|c|c|c|}
\hline Qüinqüênio & Amostra & Média & D. P. \\
\hline \multicolumn{4}{|l|}{ Sul } \\
\hline $40-44$ & 23 & 4,3 & 2,8 \\
\hline $45-49$ & 21 & 6,0 & 2,5 \\
\hline $50-54$ & 91 & 6,5 & 2,3 \\
\hline $55-59$ & 128 & 7,8 & 1,8 \\
\hline $60-65$ & 128 & 7,7 & 3,0 \\
\hline \multicolumn{4}{|l|}{ Sudeste } \\
\hline $40-44$ & 60 & 3,6 & 2,7 \\
\hline $45-49$ & 42 & 5,3 & 2,4 \\
\hline $50-54$ & 237 & 5,7 & 2,3 \\
\hline $55-59$ & 318 & 6,9 & 2,4 \\
\hline $60-65$ & 242 & 8,2 & 1,9 \\
\hline \multicolumn{4}{|c|}{ Centro-O este } \\
\hline $40-44$ & 17 & 4,1 & 2,6 \\
\hline $45-49$ & 18 & 4,4 & 2,3 \\
\hline $50-54$ & 71 & 5,5 & 2,4 \\
\hline $55-59$ & 72 & 7,6 & 2,1 \\
\hline $60-65$ & 135 & 7,4 & 2,5 \\
\hline \multicolumn{4}{|l|}{ Nordeste } \\
\hline $40-44$ & 101 & 3,7 & 2,3 \\
\hline $45-49$ & 79 & 5,3 & 2,2 \\
\hline $50-54$ & 256 & 6,0 & 2,3 \\
\hline $55-59$ & 276 & 6,9 & 2,3 \\
\hline $60-65$ & 255 & 8,2 & 1,7 \\
\hline \multicolumn{4}{|l|}{ Norte } \\
\hline $40-44$ & 10 & 4,4 & 2,8 \\
\hline $45-49$ & 30 & 6,9 & 2,5 \\
\hline $50-54$ & 144 & 7,3 & 1,9 \\
\hline $55-59$ & 186 & 8,4 & 1,7 \\
\hline $60-65$ & 152 & 8,6 & 1,9 \\
\hline \multicolumn{4}{|l|}{ Brasil } \\
\hline $40-44$ & 211 & 3,8 & 2,5 \\
\hline $45-49$ & 190 & 5,5 & 2,4 \\
\hline $50-54$ & 799 & 6,2 & 2,3 \\
\hline $55-59$ & 980 & 7,4 & 2,2 \\
\hline $60-65$ & 912 & 8,1 & 2,1 \\
\hline
\end{tabular}

dia verificada no presente estudo $(0,12 \mathrm{~cm} /$ ano) é relativamente próxima daquelas reportadas para os Municípios de Pelotas $(0,10 \mathrm{~cm} /$ ano), Bagé $(0,08 \mathrm{~cm} /$ ano $)$ e Rio Grande $(0,15$ $\mathrm{cm} / \mathrm{ano}$ ).

É interessante o fato que se refere ao efeito da Segunda Guerra Mundial. Os achados de Tanner (1966) evidenciaram uma desaceleração no crescimento por causa dos efeitos da guerra em alguns países, sobretudo da Europa (Valastovsky, 1966) e no Japão (Takahashi, 1966). No presente estudo, observou-se uma diminuição na estatura entre o primeiro e segundo qüinqüênio para o Brasil como um todo e em todas as regiões, à exceção da região Sudeste. Esta queda nas médias de estatura pode ser atribuída aos efeitos da Segunda Guerra Mundial, já que a influência exercida pelo ambiente na determinação da estatura adulta ocorre principalmente nos primeiros anos de vida (Waterlow, 1988; Tanner, 1989). É importante que estes achados sejam relativizados quando discutida a situação brasileira, na medida em que tanto os países da Europa, como o Japão, estiveram envolvidos diretamente na guerra.

Mesmo não tendo estado envolvido diretamente na guerra, está claro que a conjuntura econômica foi desfavorável ao Brasil na época. Segundo dados de Oliveira \& Lobo (1992), a inflação que havia sido de apenas 39\% entre 1929 e 1939 , elevou-se para $129 \%$ no período de guerra, tendo decaído para 86\% entre 1940 e 1947. Outra informação relevante refere-se à alta dos preços de 19 gêneros alimentícios básicos, que havia sido de $11 \%$ ao ano entre 1935 e 1938, tendo disparado para $103 \%$ entre dezembro de 1940 e novembro de 1944. Estes dados reforçam a hipótese apresentada no parágrafo anterior.

Em resumo, os achados deste trabalho demonstram a presença do fenômeno de tendência secular em estatura na amostra estudada. Ademais, recrutas com maior escolaridade, ou seja, de melhor status sócio-econômico, apresentaram as maiores taxas de ganho. Verificouse ainda que a tendência observada neste período foi menos intensa do que a reportada para a década de 70. Quando se realizou controle para a escolaridade em nível nacional, a tendência permaneceu; todavia, as reduções encontradas em todos os coeficientes demonstraram que os ganhos em estatura estiveram associados à seleção de recrutas com maior escolaridade. Por fim, argumentou-se que dados de estatura de recrutas de instituições militares utilizados para caracterizar tendência secular em estatura devem ser vistos com cautela, e que os resultados encontrados não podem ser diretamente extrapolados para a população brasileira em face das diferenças existentes nas características antropométricas e sócio-econômicas entre recrutas e a população em geral. 


\section{Agradecimentos}

O autor agradece à Diretoria de Pessoal Militar da Marinha o banco de dados colocado à disposição. Também a Célia Landman (ENSP/FIOCRUZ), Ricardo Ventura Santos (ENSP/Fiocruz e Museu Nacional/ UFRJ) e Luiz dos Anjos (UFF/Fiocruz) as diversas sugestões no desenvolvimento da investigação que resultou neste trabalho.

\section{Referências}

DEAN, A. G.; DEAN, J. A.; BURTON, A. H. \& DICKER, R. C., 1995. Epi Info, Version 6.01: a World Processing, Database, and Statistics Program for Epidemiology on Micro-Computers. Atlanta: Center for Disease Control.

EVELETH, P. B. \& TANNER, J. M., 1990. Worldwide Variation in Human Growth. Cambridge: Cambridge University Press.

FIgUeIRÓ, A. C., 1994. Perfil do Crescimento dos Jovens Alistados no Exército no Estado de Pernambuco entre 1979 e 1990. Dissertação de Mestrado, Recife: Departamento de Nutrição, Universidade Federal de Pernambuco.

FLOUD, R.; WACHTER, K. \& GREGORY, A., 1990. Height, Health and History. Nutritional Status in the United Kingdom, 1750-1980. Cambridge: Cambridge University Press.

FOGEL, W. R.; ENGERMAN, S. L. \& TRUSSEL, J., 1982. Exploring the uses of data on height: the analysis of long-term trends in nutrition, labor welfare, and labor productivity. Social Science History, 6:401-421.

FOGEL, R. W.; ENGERMAN, S. L.; FLOUD, R.; FRIEDMAN, G.; MARGO, R. A.; SOKOLOFF, K. L.; STECKEL, R. H.; TRUSSEL, J.; VILLAFLOR, G. \& WATCH$E R, W ., 1983$. Secular changes in american and british stature and nutrition. Journal of Interdisciplinary History, 15:445-481.

FOGEL, R. W., 1986. Physical growth as a measure of the economic well-being of populations: the eighteenth and nineteenth centuries. In: Human Growth. A Comprehensive Treatise. Methodology, Ecological, Genetic and Nutritional Effects on Growth (F. Falkner \& J. M. Tanner, eds.), 2nd ed., pp. 263-281, New York: Plenum Press.

GARN, S. M., 1987. The secular trend in size and maturational timing and its implications for nutritional assessment. Journal of Nutrition, 117:817823.

GREINER, T. M. \& GORDON, C. C., 1992. Secular trends of 22 body dimensions in four racial/cultural groups of american males. American Journal of Human Biology, 4:235-246.

GREULICH, W. W., 1976. Some secular changes in the growth of American-Born and native japanese children. American Journal of Physical Anthropology, 45:553-568.
IBGE (Instituto Brasileiro de Geografia e Estatística), 1963. Situação social, melhoramentos urbanos, assistência médico-sanitária. In: Anuário Estatístico do Brasil (IBGE, org.), pp. 276-277, Brasília: IBGE.

KAC, G. \& SANTOS, R.V., 1997. Secular trend in height in enlisted men and recruits from the Brazilian Navy born from 1970 to 1977. Cadernos de Saúde Pública, 3:479-487.

MALINA, R. M., 1990. Reaserch on secular trends in auxology. Anthropol gischer Anzeiger, 48:209-227.

MARCONDES, E. \& MARQUES, R. M., 1993. Estudo antropométrico de indivíduos aptos para o serviço militar no período de 1979 a 1991. Revista Brasileira de Crescimento e Desenvolvimento Humano, 2:9-38.

MARIJA, J. N., 1992. SPSS/PC+, Version 5.0. Base System User's Guide. Chicago: SPSS Inc.

MONTEIRO, C. A.; BENÍCIO, M. H. D. \& GOUVEIA, N. C., 1994. Secular growth trends in Brazil over three decades. Annals of Human Biology, 2:381390.

MONTEIRO, C. A.; BENÍCIO, M. H. D. \& GOUVEIA, N. C., 1995. Evolução da altura dos brasileiros. In: Vel hos e Novos Males da Saúde no Brasil. A Evolução do País e de suas Doenças (C. A. Monteiro, org.), pp. 126-140, São Paulo: Hucitec/ Nupens/ Universidade de São Paulo.

OLIVEIRA, A. \& LOBO, E. M. L., 1992. O Estado Novo e o sindicato corporativista, 1937-1945. In: Rio de Janeiro Operário: Natureza do Estado, a Conjuntura Econômica, Condições deVida e Consciência deClasse, 1930-1970 (E. M. L. Lobo, org.), pp. 102127, Rio de Janeiro: Acess.

PAIVA, F. C. F., 1994. Estudo da Tendência de Crescimento dos Jovens Nasci dos entre 1948 e 1974, Alistados no Serviço Militar em Rio Branco - Acre. Monografia, Rio Branco: Faculdade de Biologia, Departamento de Ciências da Natureza, Universidade Federal do Acre.

ROCHE, A. F., 1979. Secular trends in human growth, maturation, and development. Monographs of the Soci ety for Research in Child Development, 44:343.

SIM ÕES, C. C. \& MONTEIRO, C. A., 1995. Tendência secular e diferenciais regionais da mortalidade infantil no Brasil. In: Velhos e Novos Males da Saú- 
deno Brasil . A Evolução do País esuas Doenças (C. A. Monteiro, org.), pp. 153-156, São Paulo: Hucitec/ Nupens/Universidade de São Paulo.

TANNER, J. M., 1966. The secular trend towards earlier physical maturation. Tijdschrift Voor Sociale Geneeskunde, 44:524-538.

TANNER, J. M., 1981. A History of the Study of Human Growth. Cambridge: Cambridge University Press.

TANNER, J. M., 1982. The potencial of auxological data for monitoring economic and social well-being. Social ScienceHistory, 6:571-581.

TANNER, J. M., 1989. Fetus into Man. From Conception to Maturity. Cambridge: Harvard University Press.

TAKAHASHI, E., 1966. Growth and environmental factors in Japan. Human Biology, 38:112-130.
VALASTOVSKY, V. G., 1966. The secular trend in the growth and development of children and young persons in the Soviet Union. Human Biology, 38: 219-230.

VAN WIERINGEN, J. C., 1986. Secular growth changes. In: Human Growth. A Comprehensive Treatise. Methodology, Ecological, Genetic and Nutritional Effects on Growth (F. Falkner \&J. M. Tanner, eds.), pp. 307-331, New York: Plenum Press.

VICTORA, C. G.; HORTA, L. B.; RAMOS, E. O. \& CARNIELETTO, G. E., 1989. Tendência secular ao crescimento em recrutas gaúchos, 1940-1969. Ciência eCultura, 41:915-919.

WATERLOW, J. C., 1988. Observations on the natural history of stunting. In: Linear Growth Retardation in Less Devel oped Contries (J. C. Waterlow, ed.), pp. 1-16, New York: Nestlé Nutrition Workshop Series no 14. 